\title{
Outcome of Ahmed Glaucoma Valve in Refractory Glaucoma - Our Population Analysis
}

\author{
Saadia Farooq ${ }^{1}$, Momina Faisal ${ }^{2}$, Hajra Faroog ${ }^{3}$ \\ Department of Ophthalmology, ${ }^{1}$ Shifa International Hospital, Islamabad, ${ }^{2,3}$ Agha Khan University
}

\begin{abstract}
Purpose: To find out the outcomes of Ahmed Glaucoma Valve (AGV) implantation in a tertiary care hospital.

Study Design: Retrospective case series.

Place and Duration of Study: Shifa International Hospital Islamabad from January 2018 to July 2020.

Methods: This retrospective case series of 19 eyes of 17 patients with refractory glaucoma included patients who underwent AGV implantation with a follow-up period of 6 months to 01 year. AGV was done as a primary procedure in 3 patients and secondary procedure in 17 patients after a previously failed trabeculectomy. All patients underwent complete eye examination, before surgery and then at 1 month, 2months, 6 months and 1 year. Goldman tonometer was used to check IOP. Complications, interventions and the number of anti-glaucoma medications (in post-operative period) needed to achieve the target pressure were noted. Success was defined as an IOP of less than $18 \mathrm{~mm} \mathrm{Hg}$, with or without a single anti-glaucoma medication.
\end{abstract}

Results: Complete success was achieved in $57.9 \%$ and conditional success in $42.2 \%$. The most common complication was encapsulated cyst formation in $31.6 \%$ and post-operative interventions were bleb deroofing in $10.5 \%$, tube readjustment and tube wash in $10.5 \%$ and YAG vitreolysis in $5.3 \%$.

Conclusion: AGV is an effective and relatively safe procedure in refractory glaucoma irrespective of initial diagnosis and age of the patient. Encapsulated bleb was the commonest complication.

Key Words: Ahmad Glaucoma valve, Glaucoma, Intra Ocular Pressure.

How to Cite this Article: Farooq S, Faisal M. Farooq H. Outcome of Ahmed Glaucoma Valve in Refractory Glaucoma - Our Population Analysis. Pak J Ophthalmol. 2021, 37 (3): 278-282.

Doi: $10.36351 /$ pjo.v37i3.1206

\section{INTRODUCTION}

Glaucoma is the most common irreversible cause of blindness globally. ${ }^{1}$ of an estimated 79.6 million people worldwide, glaucoma prevalence was more than $50 \%$ among Asians. ${ }^{2}$.The aim of glaucoma management is to prevent progression of disease and prevent blindness by lowering IOP. This can be achieved by medications, lasers or surgical treatments. Considering the high risk of scarring in Asian eyes

Correspondence: Momina Faisal

Shifa International Hospital, Islamabad

Email: mominafaisal17@gmail.com

Received: January 16, 2021

Accepted: April 28, 2021 (which limits the success of glaucoma surgery), there is an increasing shift from simple trabeculectomy to the use of mitomycin and glaucoma drainage devices. ${ }^{3}$

Many surgical techniques are in practice for refractory glaucoma such as use of mitomycin $\mathrm{C}$, augmented trabeculectomy and the use of glaucoma drainage devices. Glaucoma drainage devices are commonly used worldwide for refractory glaucoma especially when filtering surgery has failed or there is a high risk of failure. Ahmed valve with a protective sleeve opening at pressure higher than $8 \mathrm{mmHg}$ reduces the risk of post-operative hypotony as compared to non-valve devices. ${ }^{4}$ Many studies have reported successful IOP control after AGV implant. ${ }^{3,4,5}$ The reported complications are similar to those of tube surgery i.e. tube blockage, retraction, exposure, hypotony, cataract and corneal decompensation. ${ }^{6}$ 
This study was conducted to see the efficacy, complications and visual outcome in refractory glaucoma in Al-Shifa Trust Hospital.

\section{METHODS}

We analyzed the clinical records of glaucoma patients who underwent AGV implantation at Shifa International Hospital, Islamabad, Pakistan, between January 2018 to July 2020. Ethical approval was obtained from ethical committee at Shifa International Hospital. Patient diagnosed with refractory glaucomas (primary AGV) or previous failed glaucoma surgeries (secondary AGV), IOP higher than the target with maximum topical anti-glaucoma patients and record of Minimum post-operative follow-up of greater than 6 months were included. Age less than 10 years (pediatric glaucoma) and patients with a follow-up of less than 6 months were excluded.

All surgeries were performed by a single surgeon. FP7/FP8 was implanted superotemporally under general anesthesia. $2 \%$ xylocaine with adrenaline was used to dissect conjunctiva and tenon superotemporally . FP7/ FP8 (in small hypermetropic eyes.) was primed and stitched with 8-0 proline, 8-10 $\mathrm{mm}$ from limbus. A tube was inserted in anterior chamber (phakic eyes) and ciliary sulcus (pseudophakic eyes) via entry made with 23 -gauge needle 2.5 $3 \mathrm{~mm}$ from limbus. Tube end was trimmed for not more than $3 \mathrm{~mm}$ entry in anterior Chamber with bevel up or just at pupil edge when in ciliary sulcus with bevel down. The tube was covered with partial thickness donor scleral flap stitched with 7-0 vicryl. Conjunctiva and tenon was stitched with 7-0 vicryl at limbus. Success was defined as:

Complete success- IOP 6-18 mmHg without antiglaucoma treatment and improved or stable visual acuity compared to pre-operative IOP.

Conditional success-IOP less than $18 \mathrm{mmHg}$ with 1 or 2 anti-glaucoma medications with stable visual acuity as compared to pre-operative IOP.

Failure-IOP higher than $23 \mathrm{mmHg}$ with 2 or more anti-glaucoma medications, severe co-morbids such as choroidal detachment, recurrent encysted needing revision more than 3 times.

Visual status was defined in the following terms:

Improved visual acuity; if the best corrected visual acuity improved at least one line in last follow-up.
Stable visual acuity; best corrected vision acuity remained stable.

Decreased visual acuity; best corrected vision acuity decreased at least one line or more.

Regular follow-ups were conducted on post-op day 1, 1 week, 1 month, 3 months, 6 months, 1 year and 2 years. Statistical analysis of all data was conducted using the SPSS 21. Analysis was done using t-test. A $\mathrm{P}$ value $<0.05$ was considered statistically significant. The following variables were assessed as influencing factors for surgical failure: age, gender, previous operation history, primary disease, and preoperative IOP.

\section{RESULTS}

AGV FP-7/FP-8 was performed in 19 patients. Among them 13 were male and 6 were females. Mean age was 45.167 plus minus 20.549. All patients were on maximum anti-glaucoma treatment with $84.2 \%$ having

Table 1: Prelpostoperative Vision.

\begin{tabular}{lrccc}
\hline Frequency & Percent & $\begin{array}{c}\text { Valid } \\
\text { Percent }\end{array}$ & $\begin{array}{c}\text { Cumulative } \\
\text { Percent }\end{array}$ \\
\hline$<6 / 60$ & 10 & 52.6 & 52.6 & 52.6 \\
$6 / 36-6 / 12$ & 6 & 31.6 & 31.6 & 84.2 \\
$6 / 9-6 / 6$ & 3 & 15.8 & 15.8 & 100.0 \\
Total & 19 & 100.0 & 100.0 & \\
$<6 / 60$ & 5 & 26.3 & 26.3 & 26.3 \\
$6 / 36-6 / 12$ & 9 & 47.4 & 47.4 & 73.7 \\
$6 / 9-6 / 6$ & 5 & 26.3 & 26.3 & 100.0 \\
Total & 19 & 100.0 & 100.0 & \\
\hline
\end{tabular}

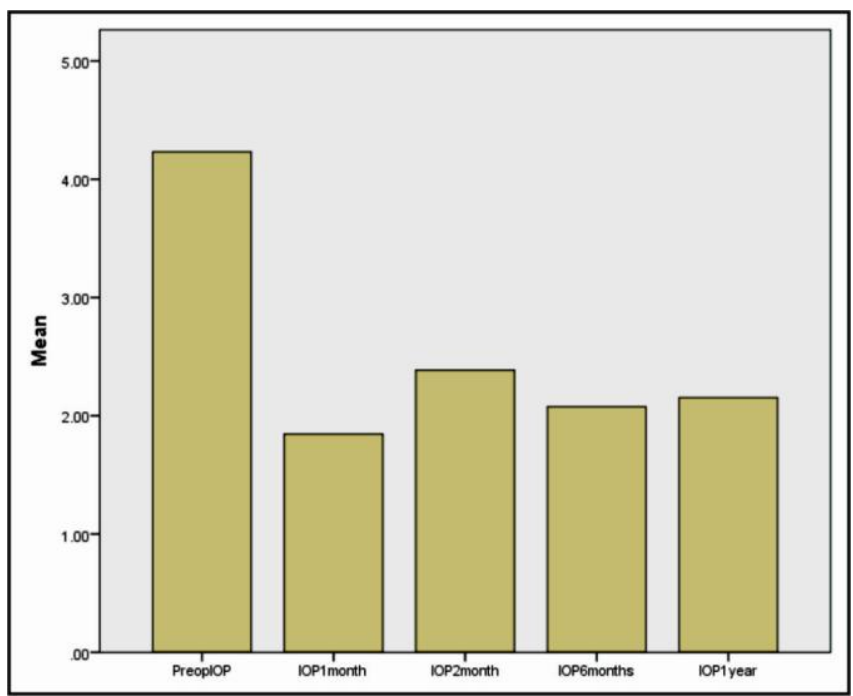

Figure 1: Post operative IOP. 
Table 2: Frequency of different glaucoma.

\begin{tabular}{llcrrr}
\hline & Frequency & Percent & Valid Percent & Cumulative Percent \\
\hline \multirow{4}{*}{ Valid } & Previous failed surgeries & 2 & 10.5 & 10.5 & 10.5 \\
& ICE syndrome & 1 & 5.3 & 5.3 & 15.8 \\
& Neurovascular glaucoma & 5 & 26.3 & 26.3 & 52.6 \\
& Angle closure glaucoma & 2 & 10.5 & 10.5 & 63.2 \\
& Post virectomy glaucoma & 5 & 26.3 & 26.3 & 89.5 \\
& Steroid induced glaucoma & 1 & 5.3 & 5.3 & 94.7 \\
& Juvenile glaucoma & 1 & 5.3 & 100.0 & 100.0 \\
& Total & 19 & 100.0 & & \\
\hline
\end{tabular}

1 or more than 1 previous surgery and $15.8 \%$ had primary valve implantation.

A total of $89.5 \%$ eyes received FP-7 implant and 10.5\% received FP-8 implant. Preoperatively 5.3\% patients had IOP in the range of range of $11-20 \mathrm{mmHg}$, whereas at 1 month the percentage increased to $73.7 \%$, at 2 months $68.4 \%$ and at 1 year $92.2 \%$ patients fell in this range. The number of medications were reduced from 4 to nil in $57.9 \%, 1$ drug in $21.1 \%$ and 2 drugs in $21.1 \%$. Complete success was achieved in $57.9 \%$ and conditional success in $42.2 \%$. The most common complication was encapsulated cyst formation in $31.6 \%$ and post-operative interventions were bleb deroofing in $10.5 \%$, tube readjustment and tube wash in $10.5 \%$ and yagvitreolysis in $5.3 \%$.

\section{DISCUSSION}

Ahmed Glaucoma Valve (FP-7 and FP-8) is a promising treatment for refractory glaucoma either in primary procedure or previous failed filtration surgeries. Overall success rate is reported from $60 \%$ to $100 \%$ at 1 year follow-up in different case series. ${ }^{7}$ Most such studies are from developed countries ${ }^{8}$, very few from Middle East. ${ }^{9,10}$

In our study $\mathrm{AGV}$ implant decreased the mean preoperative IOP from $41.58 \mathrm{mmHg}$ to $13.77 \mathrm{mmHg}$ at 1 year, reducing the number of medications from 4 to nil in $57.9 \%$. This corresponds with Zarei et al who reported mean IOP decrease from $30.8 \pm 5.6$ to $20 \pm$ $6.4 \mathrm{mmHg}$ at 4 years follow-up. ${ }^{11}$ Souza et $\mathrm{al}^{7}$ and Wishart et $\mathrm{al}^{8}$ reported significant reduction in medication after AGV implantation.

In our study Complete success was achieved in $57.9 \%$ and conditional success in $42.2 \%$, which did not vary with different types of glaucoma, age, gender or indication for surgery. This is contrary with Smith et al who reported complete success in $10.5 \%$ and guarded success in $73.7 \%{ }^{12}$ In another study increased risk of AGV failure was seen with previous incisional surgeries. ${ }^{13}$ Favorable surgical outcome is reported by Souza et $\mathrm{al}^{7}$ and Zarei et $\mathrm{al}^{11}$ reporting $50 \%$ success at 5 years and $70 \%$ at 4 consecutive years respectively. Differences in study population implants, duration of follow-up and surgical techniques could explain the differences in success rates.

Major cause of failure was fibrosis and encapsulated bleb in $31.6 \%$ of the cases, which is consistent with other reports. ${ }^{14}$ Deroofing of bleb was done in $10.5 \%$, with success in $66.7 \%$.Valimaki et al reported 52\% success and Lai et al reported $87.5 \%$ success in bleb revision. ${ }^{15,16}$ Tube erosion and implant extrusion were reported by Lai et al in $3.1 \%$ and $6.2 \%$ respectively. ${ }^{16}$ We have seen no such complication till one year follow up because scleral patch graft was used to cover the tube. Quaranta et al achieved better outcome with bovine pericardial implants to cover the valve. ${ }^{17}$ Zarei et al reported corneal decomposition in $10.7 \%$ due to tube touch. ${ }^{11}$ Where as in our study it was seen in $5.27 \%$ patients who were successfully managed by tube placement revision.

Different studies have reported hypotony varying from $9.4 \%$ to $12 \% .{ }^{18,19}$ Cataract formation is reported in $7.25 \%$ by Zareietal $^{11}$ and endophthalmitis in $6.3 \% .^{20,21}(28,29)$. No such complication was seen in our study group.

Limitation of our study is that it was a retrospective study with a small sample size and a follow-up of one year.

\section{CONCLUSION}

Ahmed Glaucoma Valve is an effective way to manage refractory glaucoma irrespective of etiology. Proper technique with posterior placement of valve, sealed conjunctival closure and short, trimmed tube in anterior chamber/posterior chamber maximally away from cornea reduces chances of unexpected 
complications. Bleb encapsulation was the most common complication affecting outcome. This probably needs more modifications like tenon excision, blunt dissection of conjunctiva and tenon with subconjunctival irrigation or use of antimetabolites.

\section{Ethical Approval}

The study was approved by the institutional review board and ethics committee, Shifa International Hospital Islamabad. (IRB\#294-1114-2020)

\section{Conflict of Interest}

Authors declared no conflict of interest.

\section{REFERENCES}

1. Toh ZH, Lee CSY, Chew ACY, Perera S. Time Heals All Wounds: Obstacles in Glaucoma Surgery from an Asian Perspective. Proc Singap Healthc. 2015; 24 (2): 103-112.

2. The number of people with glaucoma worldwide in 2010 and 2020 | Br J Ophthalmol. Available from: https://bjo.bmj.com/content/90/3/262.short

3. Elhefney E, Mokbel T, Abou Samra W, Kishk H, Mohsen T, El-Kannishy A. Long-term results of Ahmed glaucoma valve implantation in Egyptian population. Int J Ophthalmol. 2018; 11 (3): 416-421.

4. Xu Y, Hong T, Li W. Long-term outcomes of Ahmed glaucoma valve implantation for treating refractory glaucoma. Zhonghua Yi Xue Za Zhi. 2015; 95 (6): 440-443.

5. Erçalık NY, İmamoğlu S. Ahmed Glaucoma Valve Implantation in Vitrectomized Eyes. J Ophthalmol. Hindawi; Available from:

https://www.hindawi.com/journals/joph/2018/9572805/ Accessed 5, May 2020.

6. Aqueous drainage device surgery in refractory pediatric glaucomas: I. Long-term outcomes - Science Direct. Available from:

https://www.sciencedirect.com/science/article/abs/pii/S 1091853107004065. Accessed 5, May 2020

7. Souza C, Tran DH, Loman J, Law SK, Coleman AL, Caprioli J. Long-term outcomes of Ahmed glaucoma valve implantation in refractory glaucomas. Am J Ophthalmol. 2007; 144 (6): 893-900.

8. Wishart PK, Choudhary A, Wong D. Ahmed glaucoma valves in refractory glaucoma: a 7-year audit. Br J Ophthalmol. 2010; 94 (9): 1174-1179.

9. Aljazzaf AM, Abdelmoaty SMA, Behbehani AH, Abdulmuez AA, Aljazzaf HA. The outcome of the Ahmad Glaucoma Valve implantation for refractory glaucoma in Kuwait. Saudi J Ophthalmol. 2013; 27 (1): $15-18$.
10. Alasbali T, Alghamdi AA, Khandekar R. Outcomes of Ahmed valve surgery for refractory glaucoma in Dhahran, Saudi Arabia. Int J Ophthalmol. 2015; 8 (3): 560-564.

11. Zarei R, Amini H, Daneshvar R, Nabi FN, Moghimi S, Fakhraee G, et al. Long-term Outcomes of Ahmed Glaucoma Valve Implantation in Refractory Glaucoma at Farabi Eye Hospital, Tehran, Iran. Middle East Afr J Ophthalmol. 2016; 23 (1): 104-109.

12. Smith M, Geffen N, Alasbali T, Rachmiel R, Buys YM, Trope GE. Outcome of Ahmed valve implantation when preoperative IOP less than $21 \mathrm{~mm}$ Hg. J Glaucoma. 2009; 18 (9): 674-678.

13. Gedde SJ, Schiffman JC, Feuer WJ, Herndon LW, Brandt JD, Budenz DL, et al. Three-year follow-up of the tube versus trabeculectomy study. Am J Ophthalmol. 2009; 148 (5): 670-684.

10. Kirwan C, O'Keefe M, Lanigan B, Mahmood U. Ahmed valve drainage implant surgery in the management of paediatric aphakic glaucoma. $\mathrm{Br} \mathrm{J}$ Ophthalmol. 2005; 89 (7): 855-858.

11. Chen TC, Bhatia LS, Walton DS. Ahmed Valve Surgery for Refractory Pediatric Glaucoma: A Report of 52 Eyes. J Pediatr Ophthalmol Strabismus, 2005; 42 (5): 274-283.

12. A one-piece drainage system for glaucoma surgery. Europe PMC. Available from: http://europepmc.org/article/MED/3477894. Accessed May 72020.

13. Lee JH, Kim SS, Hong YJ. A Clinical Study of the Ahmed valve Implant in Refractory Glaucoma. J Korean Ophthalmol Soc. 2001; 42 (7): 1003-1010.

14. Eibschitz-Tsimhoni M, Schertzer RM, Musch DC, Moroi SE. Incidence and management of encapsulated cysts following Ahmed glaucoma valve insertion. J Glaucoma, 2005; 14 (4): 276-279.

15. Välimäki J, Tuulonen A, Airaksinen PJ. Capsule excision after failed Molteno surgery. Ophthalmic Surg Lasers, 1997; 28 (5): 382-386.

16. Lai JS, Poon AS, Chua JK, Tham CC, Leung AT, Lam DS. Efficacy and safety of the Ahmed glaucoma valve implant in Chinese eyes with complicated glaucoma. Br J Ophthalmol. 2000; 84 (7): 718-721.

17. Quaranta L, Riva I, Floriani IC. Outcomes of using a sutureless bovine pericardial patch graft for Ahmed glaucoma valve implantation. Eur J Ophthalmol. 2013; 23 (5): 738-742.

18. Ayyala RS, Zurakowski D, Smith JA, Monshizadeh R, Netland PA, Richards DW, et al. A clinical study of the Ahmed glaucoma valve implant in advanced glaucoma. Ophthalmology, 1998; 105 (10): 1968-1976.

19. Topouzis F, Coleman AL, Choplin N, Bethlem MM, Hill R, Yu F, et al. Follow-up of the original cohort with the Ahmed glaucoma valve implant. Am J Ophthalmol. 1999; 128 (2): 198-204. 
20. Gedde SJ, Scott IU, Tabandeh H, Luu KK, Budenz DL, Greenfield DS, et al. Late endophthalmitis associated with glaucoma drainage implants. Ophthalmology, 2001; 108 (7): 1323-1327.

21. Rao A, Wallang B, Padhy TR, Mittal R, Sharma S. Dual infection by streptococcus and atypical mycobacteria following Ahmed glaucoma valve surgery. Semin Ophthalmol. 2013; 28 (4): 233-235.

\section{Authors' Designation and Contribution} Saadia Farooq; Consultant Ophthalmologist: Concepts, Design, Data acquisition, Manuscript preparation, Manuscript review.

Momina Faisal; MBBS Student: Literature search, Manuscript preparation, Manuscript editing, Manuscript review.

Hajra Farooq; MBBS Student: Data analysis, Statistical analysis, Manuscript preparation. 